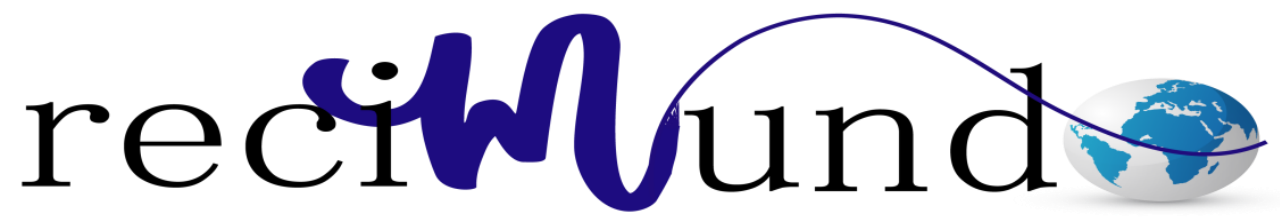

Revista Cientifica Mundo de la Investigación y el Conocimiento

Héctor Alexis Tapia Cárdenas ${ }^{\text {a }}$; Carmen Yolanda Jaramillo Calle ${ }^{\text {b }}$ Glenda

Maricela Ramón Poma ${ }^{\text {c }}$; Pedro Yamil Astudillo Arias ${ }^{\text {d}}$; Daniel Fernando Cajas Cajas ${ }^{\mathrm{e}}$

Análisis comparativo de la gestión financiera de tres Instituciones de Educación Superior del cantón Cuenca

Revista Científica Mundo de la Investigación y el Conocimiento. Vol. 2 núm., 1, febrero, ISSN: 2588-073X, 2018, pp. 550-582

DOI: 10.26820/recimundo/2.1.2018.550-582

Editorial Saberes del Conocimiento

Recibido: 05/12/2017

Aceptado: 10/02/2018

a. Universidad Católica de Cuenca.

b. Universidad Católica de Cuenca.

c. Universidad Católica de Cuenca;

d. Universidad Católica de Cuenca.

e. Universidad Católica de Cuenca; 


\section{Análisis comparativo de la gestión financiera de tres Instituciones de \\ Educación Superior del cantón Cuenca}

Vol. 2, núm. 1., (2018)

Héctor Alexis Tapia Cárdenas; Carmen Yolanda Jaramillo Calle; Glenda Maricela Ramón Poma;

Pedro Yamil Astudillo Arias; Daniel Fernando Cajas Cajas

\section{RESUMEN}

El presente trabajo presenta una visión sobre la educación, su definición e importancia además de un repaso sobre la evolución de la educación superior en el Ecuador, su marco normativo y la actual clasificación de las Instituciones de Educación Superior (IES), para luego dar paso a los principios e importancia del análisis financiero y sus principales herramientas. Finalmente se cuenta con el contexto tanto de la educación como la importancia del análisis financiero para converger en el análisis del comportamiento financiero de tres instituciones educativas, para realizar una breve comparación entre estas, tanto de los datos contables como de su gestión.

Palabras claves: IES; análisis financiero; comportamiento financiero. 


\title{
Análisis comparativo de la gestión financiera de tres Instituciones de Educación Superior del cantón Cuenca
}

Vol. 2, núm. 1., (2018)

Héctor Alexis Tapia Cárdenas; Carmen Yolanda Jaramillo Calle; Glenda Maricela Ramón Poma; Pedro Yamil Astudillo Arias; Daniel Fernando Cajas Cajas

\begin{abstract}
The present work presents a vision about education, its definition and importance as well as a review of the evolution of higher education in Ecuador, its normative framework and the current classification of Higher Education Institutions (IES), to then give way to the principles and importance of financial analysis and its main tools. Finally, we have the context of both education and the importance of financial analysis to converge in the analysis of the financial behavior of three educational institutions, to make a brief comparison between these, both accounting data and management.
\end{abstract}

Keywords: IES; financial analysis; financial behavior. 


\section{Análisis comparativo de la gestión financiera de tres Instituciones de Educación Superior del cantón Cuenca}

Vol. 2, núm. 1., (2018)

Héctor Alexis Tapia Cárdenas; Carmen Yolanda Jaramillo Calle; Glenda Maricela Ramón Poma;

Pedro Yamil Astudillo Arias; Daniel Fernando Cajas Cajas

\section{Introducción.}

En el Ecuador la Educación Superior ha tenido un cambio vertiginoso, ya que desde el año 2009 las entidades de controles iniciaron sus actividades para realizar una evaluación general a todas las instituciones de educación superior del país, para ello se debía considerar todos las normas y reglamentos que fueron creados para este fin, como es la Constitución de la República del Ecuador, la Ley Organiza de Educación Superior, el Reglamento de Régimen Académico mismos que se transformaron en las pautas que cada institución debían seguir para para cumplir con todos los requerimientos de los organismos de control.

Debemos anotar que en estos modelos de evaluación no solamente tocan los aspectos académicos sino también los administrativos y financieros, ya que al ser instituciones educativas y sin fines de lucro se debía sustentar los ingresos y los egresos que se realizaban en las universidades.

En este trabajo de investigación lo que se ha realizado es una comparación del comportamiento financiero de tres instituciones de educación superior de la ciudad de Cuenca que pasaremos a denominar Universidad A, Universidad B y Universidad C, para proteger identidades. Para ello se ha visto la necesidad de realizar un análisis de la situación de la educación superior en la actualidad y cuales han sido los cambios que se han dado y como han evolucionado estas tres instituciones en el periodo de análisis frente a los organismos de control.

Luego se ha abordado el análisis financiero cuál es su importancia en las instituciones y cuáles son los procedimientos que se siguen para determinar la situación de cada una de las 


\section{Análisis comparativo de la gestión financiera de tres Instituciones de Educación Superior del cantón Cuenca}

Vol. 2, núm. 1., (2018)

Héctor Alexis Tapia Cárdenas; Carmen Yolanda Jaramillo Calle; Glenda Maricela Ramón Poma; Pedro Yamil Astudillo Arias; Daniel Fernando Cajas Cajas

instituciones y las comparaciones que se pueden realizar entre ellas, con el fin de identificar cuáles son las fortalezas o debilidades de cada una de ellas.

Para completar con esta investigación se ha realizado la investigación a cada una de las instituciones de educación superior propuestas, con la finalidad de cumplir con los objetivos planteados en la propuesta en donde se ha realizado la comparación del comportamiento de la gestión financiera de estas universidades. Los resultados que se han obtenido de esta comparación son en primer lugar las condiciones con la que se desenvuelve cada una de ellas, este aspecto nos permite visualizar las diferencias existentes por ejemplo en los ingresos y egresos de cada una, pero lo que no se puede desconocer es la efectiva utilización de estos recursos por una de estas instituciones.

\section{La educación}

Definición e importancia

“Al alumno se le escolariza (...) para confundir enseñanza con saber, promoción al curso siguiente con educación, diploma con competencia, y fluidez con capacidad para decir algo nuevo. A su imaginación se le escolariza para que acepte servicio en vez de valor”

Iván Ilich

"La educación presupone una visión del mundo y de la vida, una concepción de la mente, del conocimiento y de una forma de pensar; una concepción de futuro y una manera de satisfacer las necesidades humanas” (Leon, 2007, pág. 598). 


\section{Análisis comparativo de la gestión financiera de tres Instituciones de Educación Superior del cantón Cuenca}

Vol. 2, núm. 1., (2018)

Héctor Alexis Tapia Cárdenas; Carmen Yolanda Jaramillo Calle; Glenda Maricela Ramón Poma; Pedro Yamil Astudillo Arias; Daniel Fernando Cajas Cajas

La educación es considerada como algo inherente al ser humano; es una actividad que tiene por fin formar, dirigir, conducir o desarrollar la vida humana para que esta, alcance su plenitud. La educación también es necesaria para la vida de los pueblos, de ahí que se considera que la grandeza de los pueblos se mide por el grado cultural de sus habitantes; por lo que la finalidad de la educación es preparar al hombre para la vida (León Quinde, 2014)

Además (Leon, 2007) afirma que la educación cambia porque el tiempo así lo dispone, porque de ella deviene. Ella misma se altera, cambia y se mueve de manera continua y a veces discontinua; crece y decrece, puede venir a ser y dejar de ser.

Donde se puede concluir que la educción es tan antigua como la conciencia humana y es ingénita a esta, proviene del ser humano y el bienestar del ser humano es su finalidad, mientras más desarrollada es, mayor prosperidad traerá no solo a la persona que la desarrolla sino a toda una colectividad e ira cambiando acorde a los tiempos y a los nuevos descubrimientos afirmándose o replanteándose a sí misma.

Derecho a la educación

Ahora bien, una vez que se ha conceptualizado la educación y determinado su trascendental importancia, es necesario saber si la educación se conceptúa también como un derecho de la humanidad y para ello es necesario tener en cuenta la existencia de tres grandes grupos de derechos: 


\section{Análisis comparativo de la gestión financiera de tres Instituciones de Educación Superior del cantón Cuenca}

Vol. 2, núm. 1., (2018)

Héctor Alexis Tapia Cárdenas; Carmen Yolanda Jaramillo Calle; Glenda Maricela Ramón Poma; Pedro Yamil Astudillo Arias; Daniel Fernando Cajas Cajas

1) Derechos Humanos: Son aquellos que son indispensables para la existencia humana es decir los mínimos esenciales para hacer posible la vida pero que sin embargo no están normados.

2) Derechos Fundamentales: Son los derechos humanos más otros adicionales que se norman y se protegen, por citar un ejemplo la Convención Americana de Derechos Humanos entre otros organismos internacionales.

3) Derechos Constitucionales: Estos son recogidos por la constitución en nuestro caso la ecuatoriana donde se norman los derechos humanos, fundamentales y otros que la constitución cree necesario proteger y así determinar las garantías constitucionales para evitar la vulneración de dichos derechos.

El núcleo esencial del derecho a la educación lo encontramos:

Disponibilidad.- Podemos definirla como las condiciones para que funcionen, dependen de numerosos factores, entre otros, el contexto de desarrollo en el que actúan; por ejemplo, las instituciones y los programas probablemente necesiten edificios u otra protección contra los elementos, instalaciones sanitarias para ambos sexos, agua potable, docentes calificados con salarios competitivos, materiales de enseñanza, entre otros; algunos necesitarán además bibliotecas, servicios de informática, tecnología de la información, etc. (Muñoz, 2012, pág. 8).

Accesibilidad. - Las instituciones y los programas de enseñanza han de ser accesibles a todos, sin discriminación: 


\section{Análisis comparativo de la gestión financiera de tres Instituciones de \\ Educación Superior del cantón Cuenca}

Vol. 2, núm. 1., (2018)

Héctor Alexis Tapia Cárdenas; Carmen Yolanda Jaramillo Calle; Glenda Maricela Ramón Poma;

Pedro Yamil Astudillo Arias; Daniel Fernando Cajas Cajas

La accesibilidad consta de tres dimensiones que coinciden parcialmente:

a. No discriminación. La educación debe ser accesible a todos, especialmente a los grupos vulnerables de hecho y de derecho, sin discriminación por ninguno de los motivos prohibidos.

b. Accesibilidad material. La educación ha de ser asequible materialmente, ya sea por su localización geográfica de acceso razonable (por ejemplo, una escuela vecinal), o por medio de la tecnología moderna (mediante el acceso a programas de educación a distancia).

c. Accesibilidad económica. La educación ha de estar al alcance de todos, se pide a los Estados que implanten gradualmente la enseñanza secundaria y superior gratuita. (Muñoz, 2012, pág. 11)

Evaluación de las instituciones de educación superior

La educación superior en el Ecuador a lo largo de su historia estuvo regida por leyes y reglamentos, pero no fue hasta la constitución del 2008 donde se dará un profundo cambio dentro de esta, dando un verdadero énfasis tanto al sistema de educación superior como a sus entes reguladores y posteriormente con la reforma de la ley Orgánica de Educación Superior(LOES) donde se determina las definitivas en su marco jurídico.

Evaluación 2009 


\section{Análisis comparativo de la gestión financiera de tres Instituciones de Educación Superior del cantón Cuenca}

Vol. 2, núm. 1., (2018)

Héctor Alexis Tapia Cárdenas; Carmen Yolanda Jaramillo Calle; Glenda Maricela Ramón Poma; Pedro Yamil Astudillo Arias; Daniel Fernando Cajas Cajas

En el 2009 se dio la primera evaluación de las Instituciones de Educación Superior (IES).

Tras el Mandato Constituyente No. 14, expedido por la Asamblea Nacional Constituyente el 22 de julio de 2008, donde se establece la obligación del Consejo Nacional de Evaluación y Acreditación (CONEA), de elaborar un informe técnico sobre el nivel de desempeño institucional de los establecimientos de educación superior, a fin de garantizar su calidad, las instituciones fueron agrupadas en 5 categorías nombradas alfabéticamente, estando en categoría “A” las de mayor puntuación en ámbitos de academia, investigación, vinculación, gestión administrativa entre otros dicha evaluación arrojo los siguientes resultados:

En la primera evaluación participaron 68 instituciones, ubicándose en categoría A 11 instituciones que representan el 16\%, en categoría B 9 instituciones que representan el 13\%, en categoría C se ubicaron el 19\% de las IES que corresponde a 9 instituciones, en categoría D se encuentran el $13 \%$ de las IES que corresponde a 9 instituciones y sorprendentemente el mayor porcentaje de universidades se ubicaron en la categoría E siendo un total de 26 instituciones que corresponden al $38 \%$ del total de las IES evaluadas.

\section{Evaluación 2013}

Para el 2013 se procedió a una nueva evaluación, fue realizado por el Consejo de Evaluación, Acreditación, y Aseguramiento de la Calidad (CEAACES), donde determina lo siguiente:

“conforme a los artículos 93, 94 y 95 de la Ley Orgánica de Educación Superior, ha definido el modelo de evaluación institucional considerando la complejidad del 


\section{Análisis comparativo de la gestión financiera de tres Instituciones de Educación Superior del cantón Cuenca}

Vol. 2, núm. 1., (2018)

Héctor Alexis Tapia Cárdenas; Carmen Yolanda Jaramillo Calle; Glenda Maricela Ramón Poma;

Pedro Yamil Astudillo Arias; Daniel Fernando Cajas Cajas

concepto de calidad y las características propias del sistema de educación superior ecuatoriano. Para el efecto, el CEAACES ha considerado los siguientes cinco criterios: Academia, Eficiencia Académica, Investigación, Organización e Infraestructura.” (CEAACES, 2013, pág. 1)

Para el mencionado proceso se contó con la participación de 54 instituciones, cabe destacar que para la fecha ya no se consideró la "Categoría E", pues las instituciones que se encontraban en esta instancia debían haber solventado las observaciones realizadas por los organismos de control en los plazos determinados para los mismos y ascender de categoría caso contrario se procedería a su cierre

"Si bien algunas instituciones han transitado de una categoría a otra, se debe tener presente que el modelo de evaluación del CONEA 2008 corresponde a un contexto distinto al de la evaluación actual. Existen diferencias en muchos indicadores; por ejemplo, en cuanto a la producción científica, el modelo de evaluación institucional del CEAACES considera el impacto de las revistas indexadas donde fueron publicados los artículos, mientras que en el modelo de 2008 solamente se consideró el número de publicaciones. Sin embargo, mediante indicadores comparables, se puede notar que el sistema de universidades y escuelas politécnicas del Ecuador ha mejorado" (CEAACES, 2013, pág. 2)

En la evaluación del 2013 participaron 54 instituciones, ubicándose en "categoría A" apenas 5 instituciones que representan el 9\% del total de IES evaluadas, en "categoría B" 23 instituciones que representan el $43 \%$, en la "categoría C" se ubicaron el 18\% de las IES que 


\section{Análisis comparativo de la gestión financiera de tres Instituciones de Educación Superior del cantón Cuenca}

Vol. 2, núm. 1., (2018)

Héctor Alexis Tapia Cárdenas; Carmen Yolanda Jaramillo Calle; Glenda Maricela Ramón Poma; Pedro Yamil Astudillo Arias; Daniel Fernando Cajas Cajas

corresponde a 9 instituciones, en "categoría D" se encuentran el 15\% de las IES que corresponde a 8 instituciones, para esta clasificación ya no se contó con la "categoría E” como se menciona anteriormente.

\section{Evaluación 2015}

Durante el 2015 se dió un proceso donde 12 instituciones incurrieron en la $\operatorname{Re}$ categorización voluntariamente más la Universidad de las Fuerzas Armadas (ESPE) para la cual fue obligatorio por no haber sido considerada en el proceso del 2013 (CEAACES, 2017)

El CEAACES en su portal web ha publicado de manera permanente y actualizada las IES que conforman el Sistema de Educación Superior y su categoría actual considerando casos como los de la Universidad Católica de Cuenca que se ha sometido voluntariamente al proceso de Re categorización en el 2017 y que ya consta su nueva categoría en el mencionado portal.

En la categorización obtenida al año 2017 se cuenta con 55 instituciones, ubicándose en "categoría A" apenas 8 instituciones que representan el 15\% del total de IES evaluadas, en “categoría B" 28 instituciones que representan el 51\% del total de IES evaluadas, en la “categoría C" se ubican el 35\% de las IES que corresponde a 19 instituciones, en la mencionada “categoría D” ya no se encuentran Universidades en la actualidad.

Durante los procesos de evaluación por los que han pasado la educación superior en el ecuador, se ve una mejoría según los informes emitidos por los organismos de control, pues las 


\section{Análisis comparativo de la gestión financiera de tres Instituciones de Educación Superior del cantón Cuenca}

Vol. 2, núm. 1., (2018)

Héctor Alexis Tapia Cárdenas; Carmen Yolanda Jaramillo Calle; Glenda Maricela Ramón Poma;

Pedro Yamil Astudillo Arias; Daniel Fernando Cajas Cajas

categorías inferiores han ido suprimiéndose y con ello las instituciones que se encontraban en dichos rangos se han visto obligadas a mejorar y solventar las observaciones recibidas para no ser obligadas a su cierre definitivo al cual como fueron sometidas ciertas instituciones por no cumplir las condiciones mínimas para la enseñanza universitaria, contradictoriamente también se puede evidenciar que estar en categoría A no es un garantía de por vida pues las instituciones no puede dormirse en los laureles o bajaran a categorías inferiores.

\section{Análisis financiero}

El análisis financiero busca comparar de forma sistemática todos los valores o recursos que se han movido, sean estos del activo o del pasivo, buscando en qué grado esta la compatibilidad entre estos, desde el punto de vista de la estabilidad financiera de la organización y cuales son o serán las posibilidades de desarrollo pero con equilibrio con el fin de que la toma de decisiones sean más eficientes y justo en el momento idóneo (Valls P., 1999).

\section{Importancia}

La importancia que tiene el análisis de los estados financieros, es porque son uno de los componentes más necesarios para la toma de decisiones en una empresa, por parte de los inversionistas o los interesados en conocer claramente la situación de la organización en el ámbito económico y financiero (Córdova Padilla, 2016)

La actividad financiera se puede aplicar cuando exista rigurosidad en el análisis que se efectúa previamente. A las conclusiones que se llegan en este estudio previo son muy importantes para que la toma de decisiones sea más eficiente y fácil de tomarlas y determinar con 


\section{Análisis comparativo de la gestión financiera de tres Instituciones de Educación Superior del cantón Cuenca}

Vol. 2, núm. 1., (2018)

Héctor Alexis Tapia Cárdenas; Carmen Yolanda Jaramillo Calle; Glenda Maricela Ramón Poma; Pedro Yamil Astudillo Arias; Daniel Fernando Cajas Cajas

mayor facilidad las estrategias. En el análisis financiero se toman los documentos contables, en los cuales se trabajan desde el inicio, no se debe tomar solamente de evidencia recopilada sino de un trabajo que sea realizada en conjunto siguiendo un proceso exhaustivo tomando como base la información verídica es decir tomando la realidad de la empresa y de los objetivos financieros que tenga la organización (Chavéz, 2003).

"La situación financiera internacional nos obliga a administrar adecuadamente y de forma racional los recursos materiales, financieros y humanos con vistas a lograr la eficiencia en la productividad empresarial. La evaluación de los resultados de la gestión de la empresa es el punto de partida para tomar numerosas decisiones en el proceso de administrar de manera que sean consecuentes con las tendencias de comportamiento en el futuro y que puedan predecir su situación económica y financiera, en aras del desarrollo de la economía de un país. Para lograr una correcta Administración Financiera, es necesario realizar un adecuado Análisis Económico Financiero, el representa un medio infalible para el control de los recursos y para evaluar la situación económica financiera de las entidades; le propiciará a los directivos lograr controles económicos ágiles con vistas a facilitar la toma de decisiones y a tener una administración más eficiente" (Escobar, 2013).

"Una empresa que enfrente un entorno difícil y convulsionado con los inconvenientes descritos anteriormente, debe implementar medidas que le permitan ser más competitiva y eficiente desde la perspectiva económica y financiera, de forma tal que haga mejor uso de sus recursos para obtener mayor productividad y 


\section{Análisis comparativo de la gestión financiera de tres Instituciones de Educación Superior del cantón Cuenca}

Vol. 2, núm. 1., (2018)

Héctor Alexis Tapia Cárdenas; Carmen Yolanda Jaramillo Calle; Glenda Maricela Ramón Poma;

Pedro Yamil Astudillo Arias; Daniel Fernando Cajas Cajas

mejores resultados con menores costos; razón que implica la necesidad de realizar un análisis exhaustivo de la situación económica y financiera de la actividad que lleva a cabo.” (Navas, 2009)

El análisis de los estados financieros permite evaluar el desempeño financiero y operativo de una empresa, observando tendencias, márgenes, indicadores, razones, fortalezas y debilidades en cuanto a la disponibilidad de recursos y su estructura financiera y económica...” además permite "tener y conocer todos los elementos suficientes para saber si una empresa está marchando normal y adecuadamente o no, para tomarlos correctivos necesarios a tiempo y poder salvarla de una situación financiera mala (ESTUPIÑAN \& ESTUPIÑAN, 2007, pág. 96)

En conclusión, podemos decir que el análisis financiero es de gran importancia para cualquier empresa independiente mente de la actividad a la que se dedique, pues permite determinar cuál es la situación financiera de la empresa y si esta es saludable o no; Además permite conocer el resultado de las decisiones previas y formular nuevas decisiones y estrategias a tomar, con el fin de optimizar la utilización de los recursos de la entidad.

\section{Definición}

El análisis financiero es el estudio de todos los ingresos, los cotos y la rentabilidad de las organizaciones, empresas o instituciones individuales, tomando en consideración todos los factores de producción que han sido adquiridos en los precios corrientes del mercado. Además podemos anotar que es el instrumento más importante, mismo que permite determinada cual es la 


\section{Análisis comparativo de la gestión financiera de tres Instituciones de Educación Superior del cantón Cuenca}

Vol. 2, núm. 1., (2018)

Héctor Alexis Tapia Cárdenas; Carmen Yolanda Jaramillo Calle; Glenda Maricela Ramón Poma; Pedro Yamil Astudillo Arias; Daniel Fernando Cajas Cajas

capacidad financiera que posee una empresa u organización, para llevar adelante sus actividades con normalidad y de forma eficiente (Pérez E., 1993).

Se trata de un proceso de reflexión con el fin de evaluar la situación financiera actual y pasada de la empresa, así como los resultados de sus operaciones, con el objetivo básico de determinar del mejor modo posible, una estimación sobre la situación y los resultados futuros. (Rubio, 2007, pág. 2).

El análisis financiero es el conjunto de técnicas que se utilizan con la finalidad de obtener un diagnostico real de la situación y cuáles son las perspectivas de la empresa, entonces la finalidad del análisis financiero es tener la posibilidad de tomar decisiones eficientes y adecuadas en el entorno de la empresa (Domínguez \& Olalla, 2012).

\section{Herramientas del análisis financiero}

"Uno de las herramientas para el análisis financiero son los indicadores financieros, mismos que se utilizan como herramienta indispensable ya que permite determinar su condición financiera; pues a través de su cálculo e interpretación se logra ajustar el desempeño operativo de la organización logrando así identificar aquellas áreas de mayor rendimiento y aquellas que deben ser mejoradas. Entre los ratios más están: indicadores de liquidez y solvencia, indicadores de eficiencia o actividad, indicadores de endeudamiento y los indicadores de rentabilidad". (Navas, 2009)

Dada la naturaleza de las instituciones que vamos a evaluar se citaran únicamente ciertos indicadores que son aplicables a este tipo de instituciones 


\section{Análisis comparativo de la gestión financiera de tres Instituciones de}

Educación Superior del cantón Cuenca

Vol. 2, núm. 1., (2018)

Héctor Alexis Tapia Cárdenas; Carmen Yolanda Jaramillo Calle; Glenda Maricela Ramón Poma;

Pedro Yamil Astudillo Arias; Daniel Fernando Cajas Cajas

Comparación de tres instituciones de educación superior del cantón Cuenca

A continuación, se realiza una comparación de los principales factores que incidieron en la situación financiera de las IES y se obtendrá un breve análisis de dichas comparaciones.

Figura 1. Comparación de número de estudiantes entre instituciones

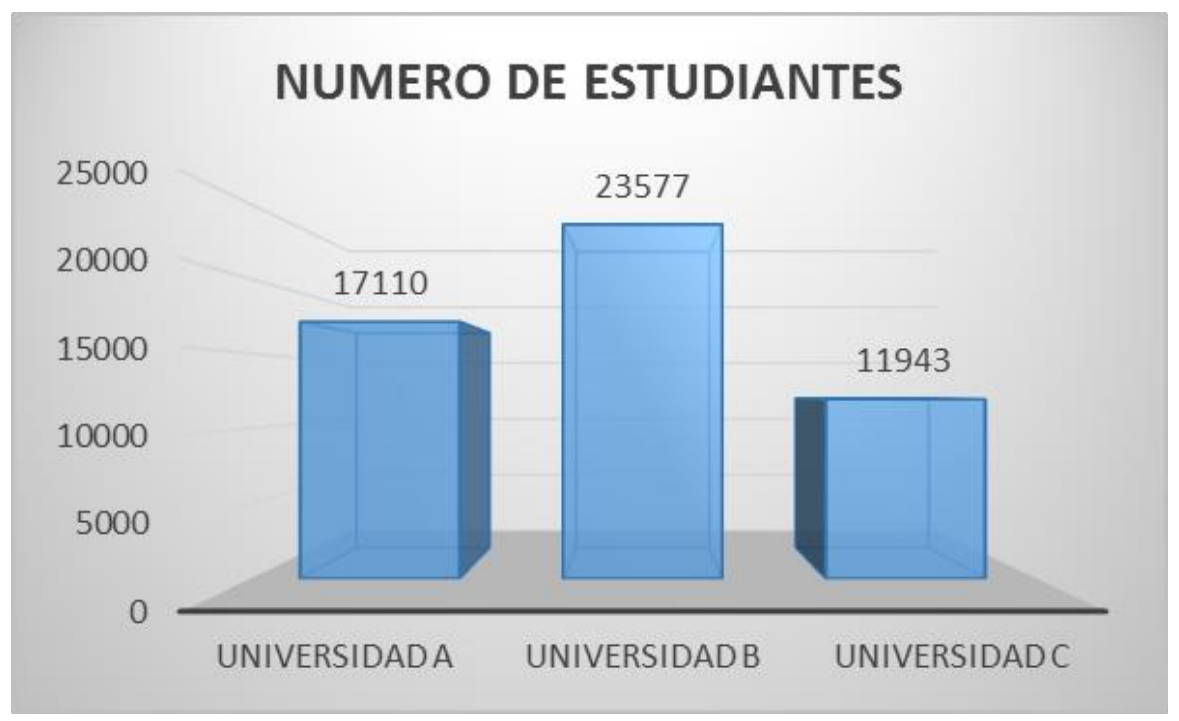

Fuente: Elaborado por los autores

Durante el desarrollo del estudio la institución que más estudiantes albergo fue la Universidad "B" mostrando una notoria superioridad en este aspecto tanto así que la institución cuenta con 37,8\% más de estudiantes que las otras dos universidades objeto de estudio. 
Análisis comparativo de la gestión financiera de tres Instituciones de Educación Superior del cantón Cuenca

Vol. 2, núm. 1., (2018)

Héctor Alexis Tapia Cárdenas; Carmen Yolanda Jaramillo Calle; Glenda Maricela Ramón Poma; Pedro Yamil Astudillo Arias; Daniel Fernando Cajas Cajas

Figura 2. Comparación de número de docentes entre instituciones

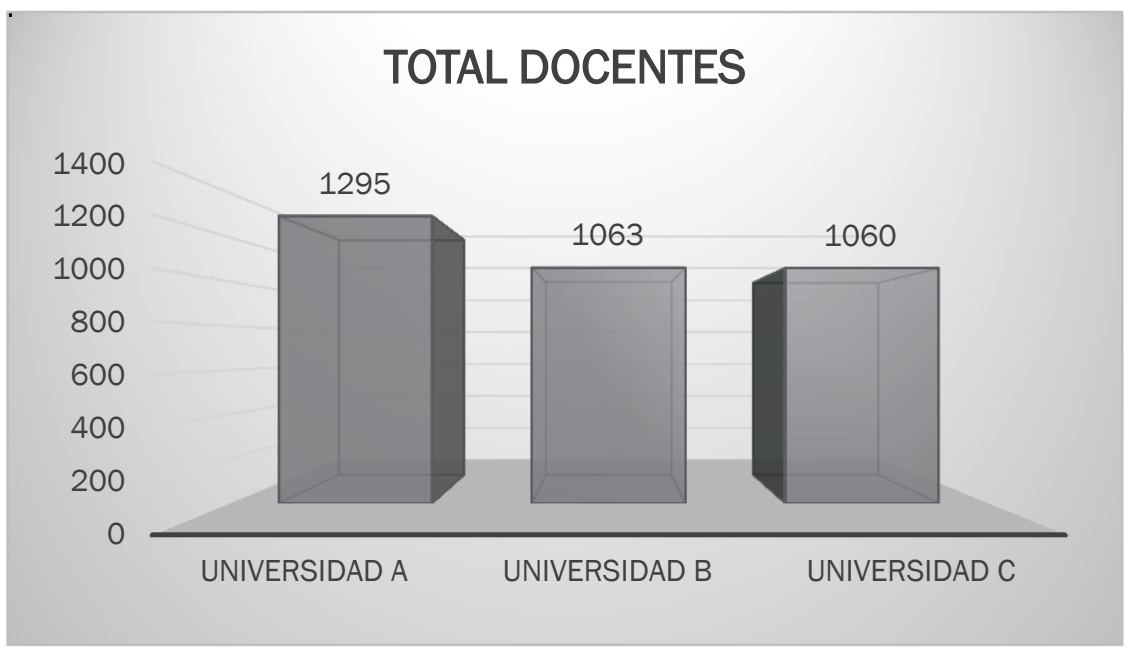

Fuente: Elaborado por los autores

Dentro de este parámetro de comparación es evidente una cierta paridad, pues todas las diferencias entre las instituciones analizadas es mínima, sin embargo una de ellas muestra una leve superioridad de la media, ahora este ratio contrasta con el número de estudiantes pues en el caso de la Universidad "C" con un número menor de los mismos, posee un cuerpo docente 


\section{Análisis comparativo de la gestión financiera de tres Instituciones de}

Educación Superior del cantón Cuenca

Vol. 2, núm. 1., (2018)

Héctor Alexis Tapia Cárdenas; Carmen Yolanda Jaramillo Calle; Glenda Maricela Ramón Poma; Pedro Yamil Astudillo Arias; Daniel Fernando Cajas Cajas

similar al resto de instituciones y para entender mejor este aspecto es necesario comparar otros aspectos complementarios como la oferta académica o el número de sedes y extensiones con los que cuenta cada institución.

Figura 3. Comparación del número de carreras entre instituciones

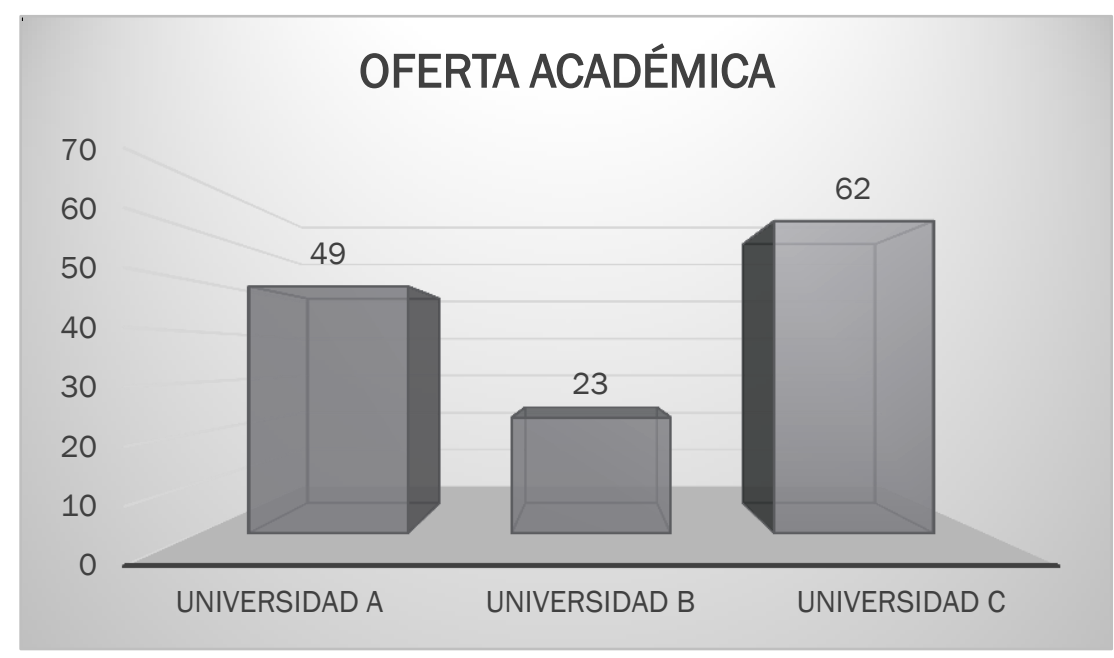

Fuente: Elaborado por los autores

La institución que más carreras activas obtuvo fue la "C", pues mantuvo un total de 62 entre matriz sedes y extensiones, superando en un $27 \%$ a la universidad " $\mathrm{A}$ " y en un $17 \%$ a la Universidad "B". 
Análisis comparativo de la gestión financiera de tres Instituciones de Educación Superior del cantón Cuenca

Vol. 2, núm. 1., (2018)

Héctor Alexis Tapia Cárdenas; Carmen Yolanda Jaramillo Calle; Glenda Maricela Ramón Poma; Pedro Yamil Astudillo Arias; Daniel Fernando Cajas Cajas

Figura 4. Comparación del número del sedes y extensiones entre instituciones

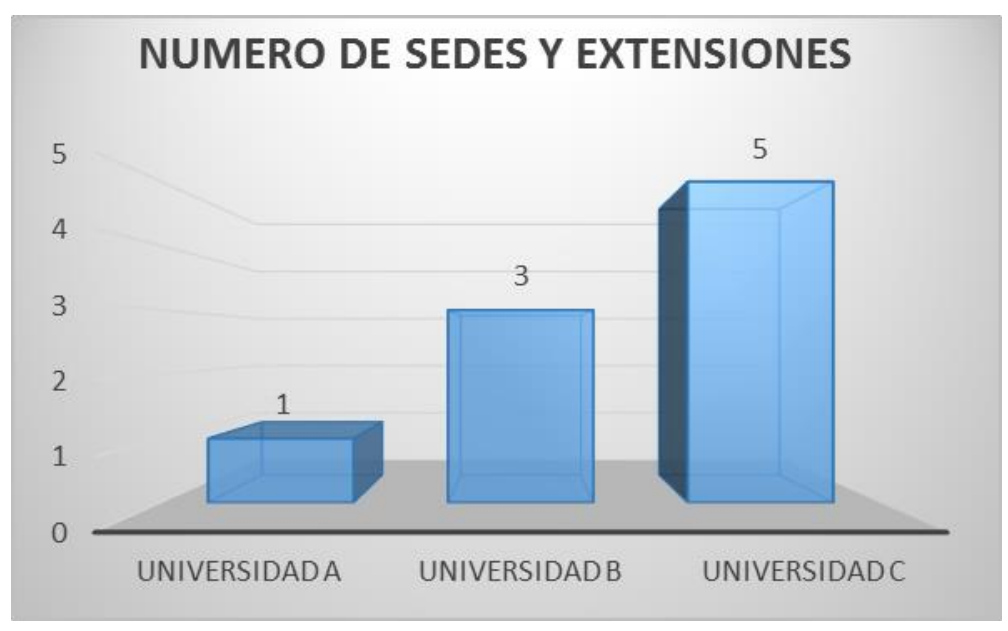

Fuente: Elaborado por los autores.

La Universidad "C" es la de mayor cobertura de las instituciones analizadas pues posee 5 dependencias entre matriz sedes y extensiones y se encuentra presente en tres provincias del país siendo estas Azuay, Cañar y Morona Santiago, mientras que la Universidad "B" pose tres 


\section{Análisis comparativo de la gestión financiera de tres Instituciones de Educación Superior del cantón Cuenca}

Vol. 2, núm. 1., (2018)

Héctor Alexis Tapia Cárdenas; Carmen Yolanda Jaramillo Calle; Glenda Maricela Ramón Poma; Pedro Yamil Astudillo Arias; Daniel Fernando Cajas Cajas

dependencias distribuidas en igual número de provincias las cuales son Azuay, Pichincha y Guayas y por último la Universidad "A" está presente únicamente en la ciudad del mismo nombre.

Con estos datos podemos tener una lectura un poco más clara de los gráficos anteriores pues nos permite tener una apreciación más adecuada de la gestión y utilización de capital humano, pues la Universidad "A" tiene un número considerable de estudiantes, todos estos concentrados únicamente en una sola sede y una sola ciudad, obviamente esto no quiere decir que sus estudiantes provengan todos de la misma ciudad en gran parte por la gratuidad de la misma, sin embargo es la que mayor cantidad de docentes posee y contando con un docente por cada 13 alumnos, mientras que en términos generales la Universidad "C" cuenta con 1 docente por cada 11 alumnos y la "B" posee un docente por cada 22 alumnos; pero se debe tener en cuenta que no son condiciones iguales, por lo que esta comparación no se puede realizar dado que las instituciones privadas deben distribuir a su planta docente en las distintas sedes que posee y de igual manera la concentración de sus alumnos no va ser la misma, por lo tanto la necesidad de docentes no será igual en cada una de las sedes y extensiones.

Otro factor a considerar es la oferta académica pues al poseer mayor variedad de carreras se necesita más profesionales en distintas áreas, ahora bien, si revisamos la información anterior la institución que más concentración tiene en sus inmuebles con relación a sus activos fue la Universidad "C", pues este representaba alrededor del $83 \%$ mientras que para la Universidad pública representa apenas el $41 \%$; es decir, menos de la mitad con relación a la Universidad privada. 
Análisis comparativo de la gestión financiera de tres Instituciones de Educación Superior del cantón Cuenca

Vol. 2, núm. 1., (2018)

Héctor Alexis Tapia Cárdenas; Carmen Yolanda Jaramillo Calle; Glenda Maricela Ramón Poma; Pedro Yamil Astudillo Arias; Daniel Fernando Cajas Cajas

Figura 5. Comparación de las transferencias recibidas entre instituciones

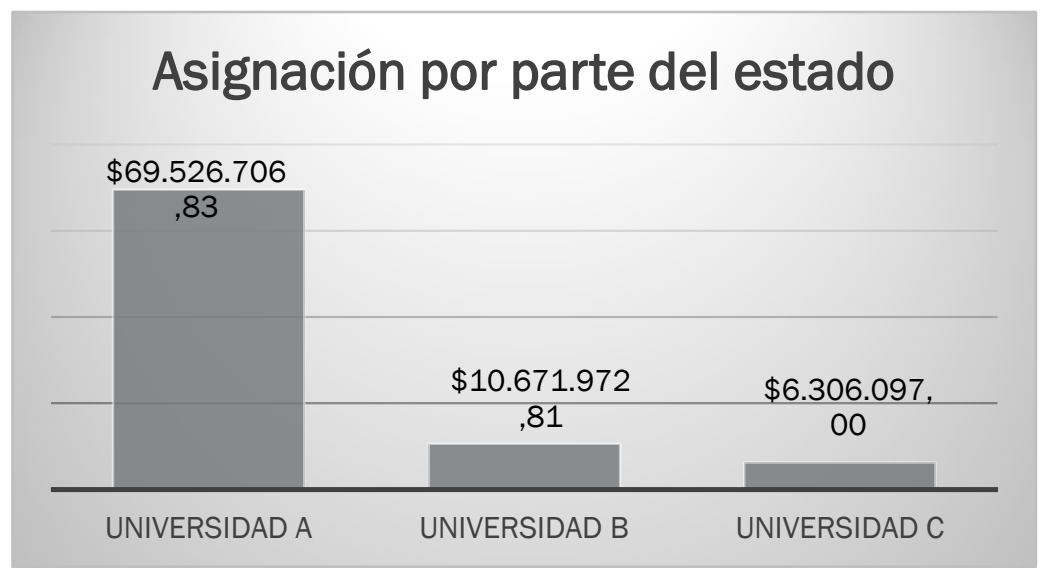

Fuente: Elaborado por los autores

Evidentemente por la naturaleza de la Universidad "A" es la que más recursos percibe por parte del estado por ser pública, mientras que por su nivel de alumnos y de acuerdo a lo 


\section{Análisis comparativo de la gestión financiera de tres Instituciones de \\ Educación Superior del cantón Cuenca}

Vol. 2, núm. 1., (2018)

Héctor Alexis Tapia Cárdenas; Carmen Yolanda Jaramillo Calle; Glenda Maricela Ramón Poma;

Pedro Yamil Astudillo Arias; Daniel Fernando Cajas Cajas

determinado por la ley la Universidad "B" es la que más recursos percibe por parte del estado por su calidad de cofinanciada y por último la Universidad "C"

Figura 6. Comparación de los ingresos correspondientes

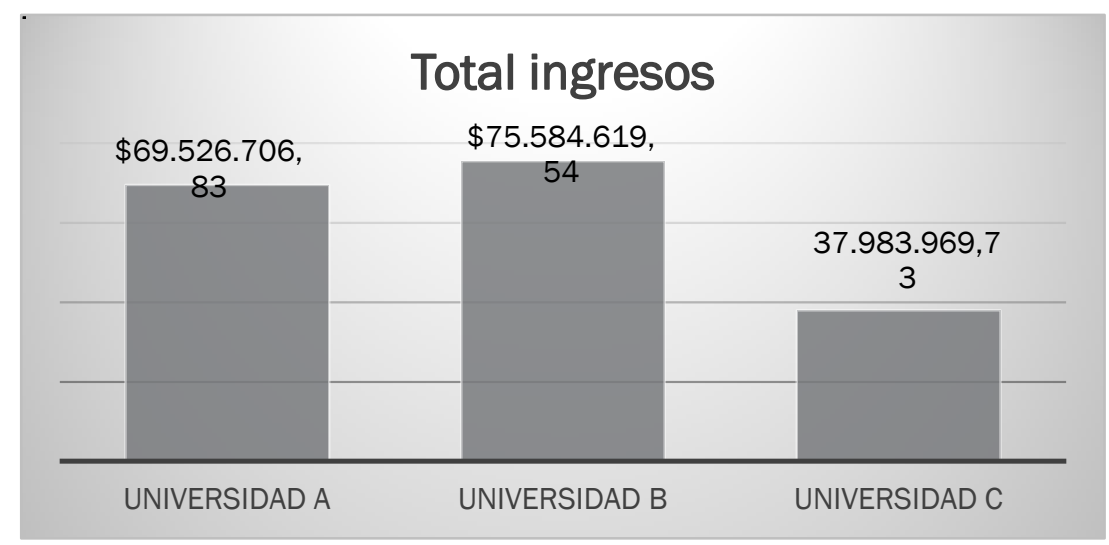

Fuente: Elaborado por los autores

La Universidad "B" es la que lidera esta comparación de total de ingresos, pues con relación a la Universidad "A" es del $8.71 \%$, mientras que en comparación con la Universidad "C" es del $98.99 \%$, diferencia que se da por el número de estudiantes, principal fuente de ingresos para estas dos instituciones, para el año de comparación la diferencia de estudiantes fue del $97.41 \%$ a esto si consideramos el rubro de asignaciones por parte del estado que, es otro de los factores más significativos en esta comparación en la que se existe una diferencia de 4,365,875.81 de dólares entre la "B" y la "C".

Figura 7. Comparación de las utilidades 
Análisis comparativo de la gestión financiera de tres Instituciones de Educación Superior del cantón Cuenca

Vol. 2, núm. 1., (2018)

Héctor Alexis Tapia Cárdenas; Carmen Yolanda Jaramillo Calle; Glenda Maricela Ramón Poma; Pedro Yamil Astudillo Arias; Daniel Fernando Cajas Cajas

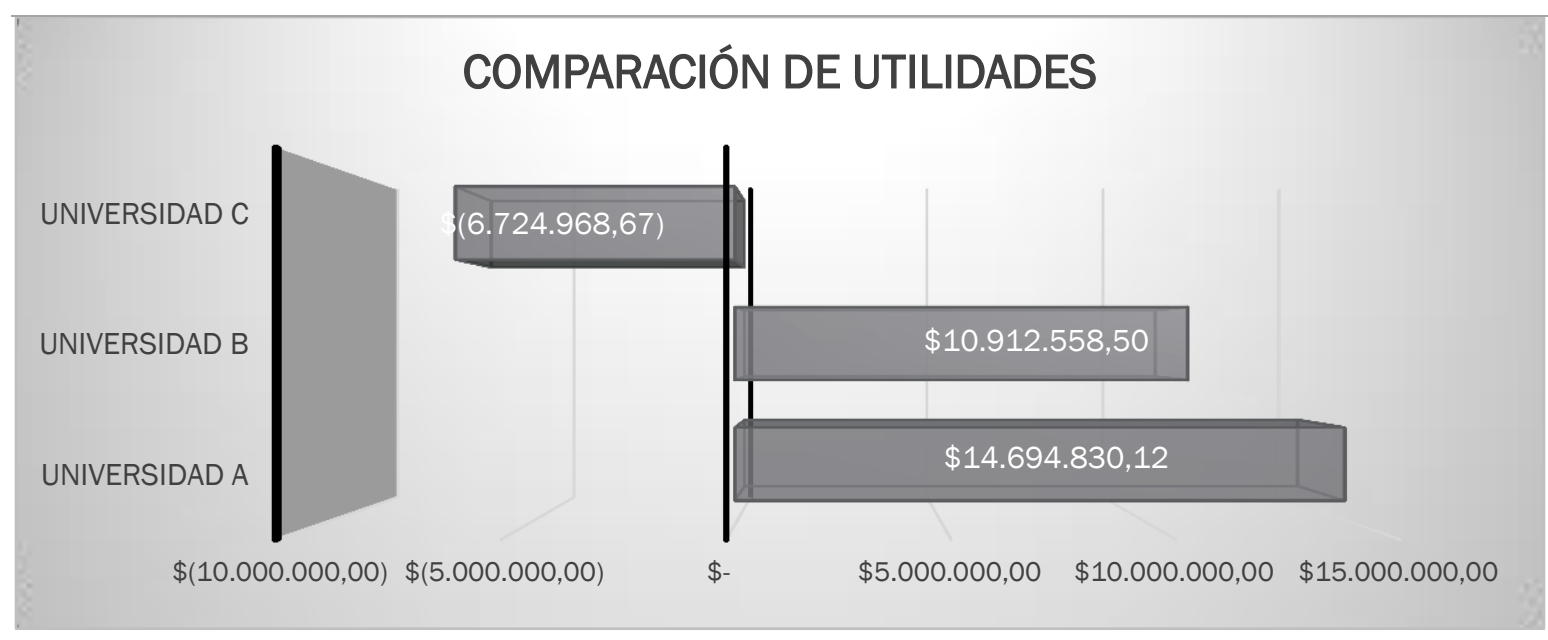

Fuente: Elaborado por los autores

Las utilidades de estas instituciones tiene un contraste con sus ingresos pues como podemos ver, la que tiene más ingresos no necesariamente será la que más utilidad tenga, sino la que más eficiente es con los mismos, para este caso la Universidad pública es la que mayor utilidad obtuvo mientras que en lado opuesto la Universidad "C" es la de mayor preocupación con una perdida bastante considerable y más aún, cuando esta ha sido creciente en los últimos tres años como se pudo evidenciar en la comparación de su utilidades que se realizó anteriormente.

Figura 8. Comparación del patrimonio entre instituciones 


\section{Análisis comparativo de la gestión financiera de tres Instituciones de}

Educación Superior del cantón Cuenca

Vol. 2, núm. 1., (2018)

Héctor Alexis Tapia Cárdenas; Carmen Yolanda Jaramillo Calle; Glenda Maricela Ramón Poma; Pedro Yamil Astudillo Arias; Daniel Fernando Cajas Cajas

\section{Comparación del Patrimonio}

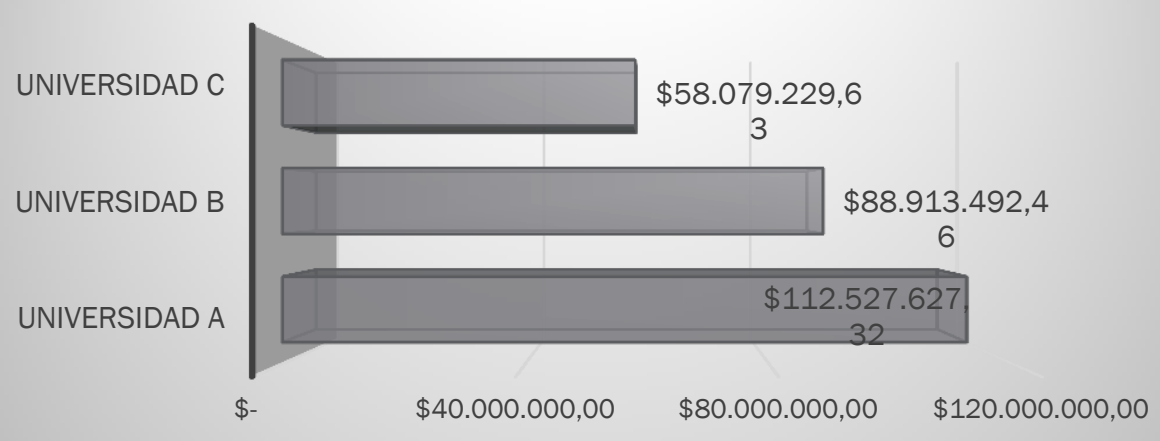

Fuente: Elaborado por los autores.

La institución que presenta un mayor volumen en sus patrimonios es la universidad pública con un patrimonio muy por encima de sus similares con una relación de $26.56 \%$ más que la Universidad "B" y $93.75 \%$ más que la universidad "C".

Comparación de ratios financieros

Figura 3. Comparación de la razón corriente entre instituciones 
Análisis comparativo de la gestión financiera de tres Instituciones de Educación Superior del cantón Cuenca

Vol. 2, núm. 1., (2018)

Héctor Alexis Tapia Cárdenas; Carmen Yolanda Jaramillo Calle; Glenda Maricela Ramón Poma; Pedro Yamil Astudillo Arias; Daniel Fernando Cajas Cajas

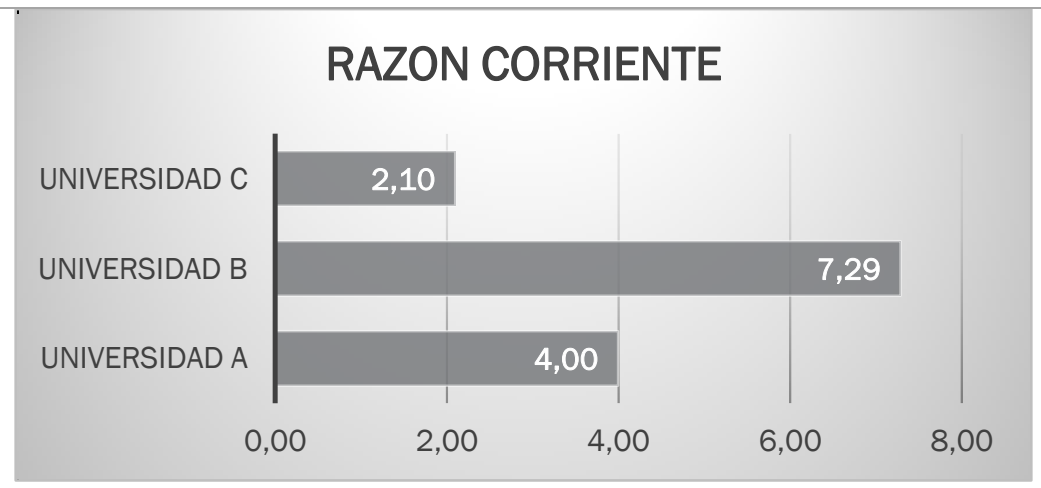

Fuente: Elaborado por los autores

La Universidad que más alto ratio presenta es la Universidad B, sin embargo, esto demuestra que dicha institución posee capitales inactivos que pudiesen generar mayor beneficio a la institución; por su parte tanto la Universidad C como la Universidad A muestran ratios positivos que permiten ver que pueden hacer frente a sus obligaciones a corto plazo.

\section{Figura 4. Comparación de la prueba ácida entre instituciones}

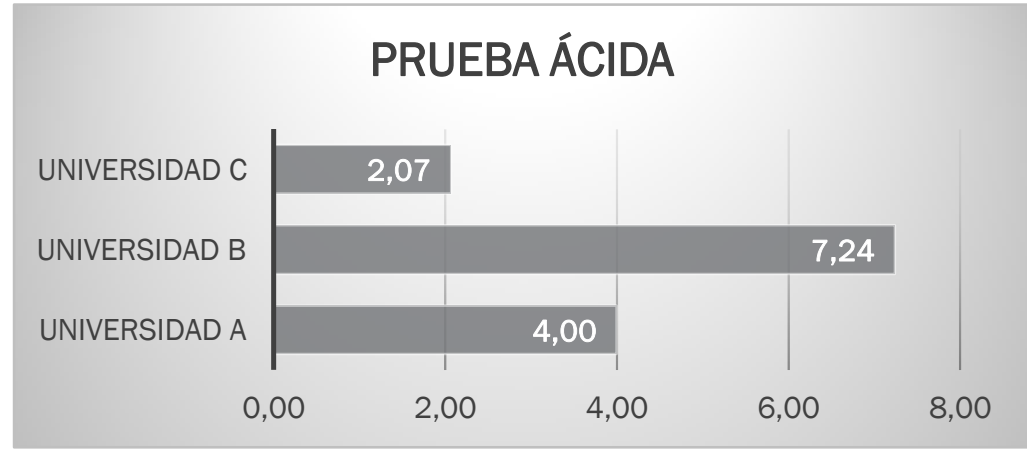

Fuente: Elaborado por los autores

Este ratio presenta las mismas características que el anterior pues involucra a las mismas cuentas contables, sin embargo se debe restar los inventarios del activo corriente para obtener un 


\section{Análisis comparativo de la gestión financiera de tres Instituciones de Educación Superior del cantón Cuenca}

Vol. 2, núm. 1., (2018)

Héctor Alexis Tapia Cárdenas; Carmen Yolanda Jaramillo Calle; Glenda Maricela Ramón Poma;

Pedro Yamil Astudillo Arias; Daniel Fernando Cajas Cajas

resultado más acertado, pero dada la naturaleza de las instituciones educativas los inventarios son mínimos o en ciertos casos nulos según sus estados financieros por lo que, los valores cambian únicamente en decimales y por lo tanto se podría dar la misma interpretación que en ratio anterior, pues todas las instituciones pueden hacer frente a sus obligaciones a corto plazo sin inconvenientes.

Figura 5. Comparación de la independencia financiera entre instituciones

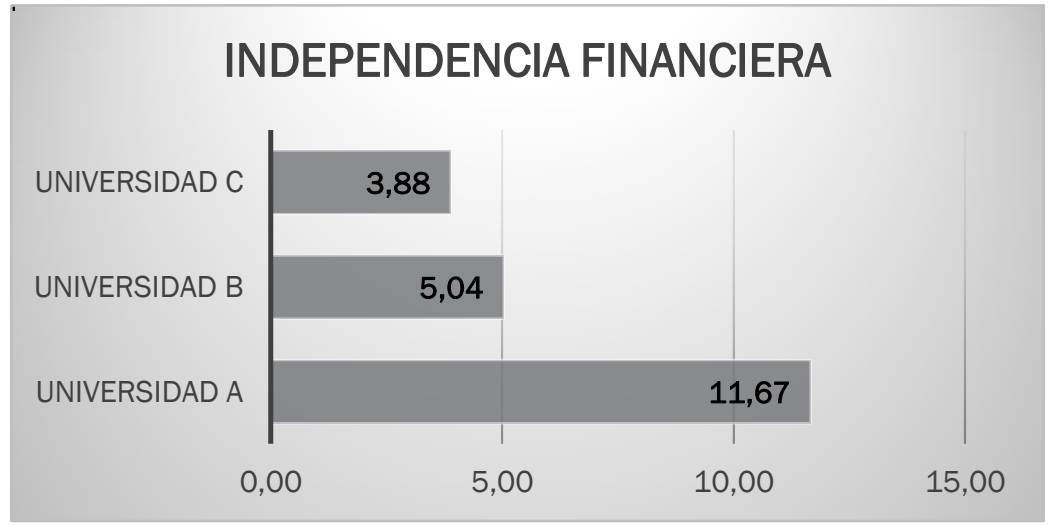

Fuente: Elaborado por el autor.

El presente indicador se utiliza para medir la relación de capitales propios con los capitales ajenos; es decir, en el caso de la Universidad A, por ejemplo, por cada dólar de deuda la institución posee 11 dólares con 67 centavos en su patrimonio, ahora bien, esto refleja que las instituciones poseen un capital amplio con relación a sus deudas, ósea que su nivel de endeudamiento no supera a sus patrimonios y poseen independencia financiera, unas instituciones más que otras.

Figura 6. Comparación del capital de trabajo entre instituciones 


\section{Análisis comparativo de la gestión financiera de tres Instituciones de}

Educación Superior del cantón Cuenca

Vol. 2, núm. 1., (2018)

Héctor Alexis Tapia Cárdenas; Carmen Yolanda Jaramillo Calle; Glenda Maricela Ramón Poma; Pedro Yamil Astudillo Arias; Daniel Fernando Cajas Cajas

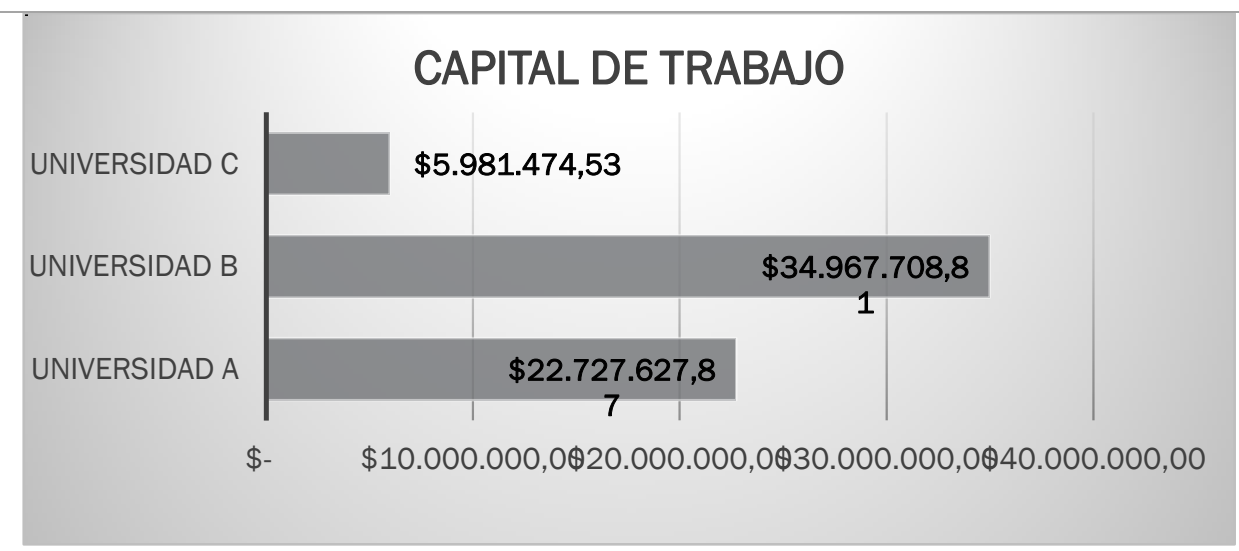

Fuente: Elaborado por el autor.

El capital de trabajo es el factor que permite que la institución siga funcionando normalmente, en este punto vemos que la Universidad B supera con mucho al resto de instituciones exactamente 54\% más que la Universidad A y $485 \%$ más que la Universidad C.

Por otro lado podemos confirmar lo que ya se interpretaba en ratios anteriores en cuanto al exceso de liquidez de la Institución B pues debemos tomar en cuenta que por la naturaleza de las instituciones educativas el inventario representa un porcentaje menor del capital de trabajo, pues el total de las deudas a corto plazo representan $\$ 5,560,399.23$ de dólares y mientras que el disponible de la institución específicamente en caja y bancos es de $\$ 12,165,853.38$ y estos representan únicamente el $11 \%$ con relación al total de sus activos.

Figura 7. Comparación del índice de endeudamiento entre instituciones 


\section{Análisis comparativo de la gestión financiera de tres Instituciones de Educación Superior del cantón Cuenca}

Vol. 2, núm. 1., (2018)

Héctor Alexis Tapia Cárdenas; Carmen Yolanda Jaramillo Calle; Glenda Maricela Ramón Poma; Pedro Yamil Astudillo Arias; Daniel Fernando Cajas Cajas

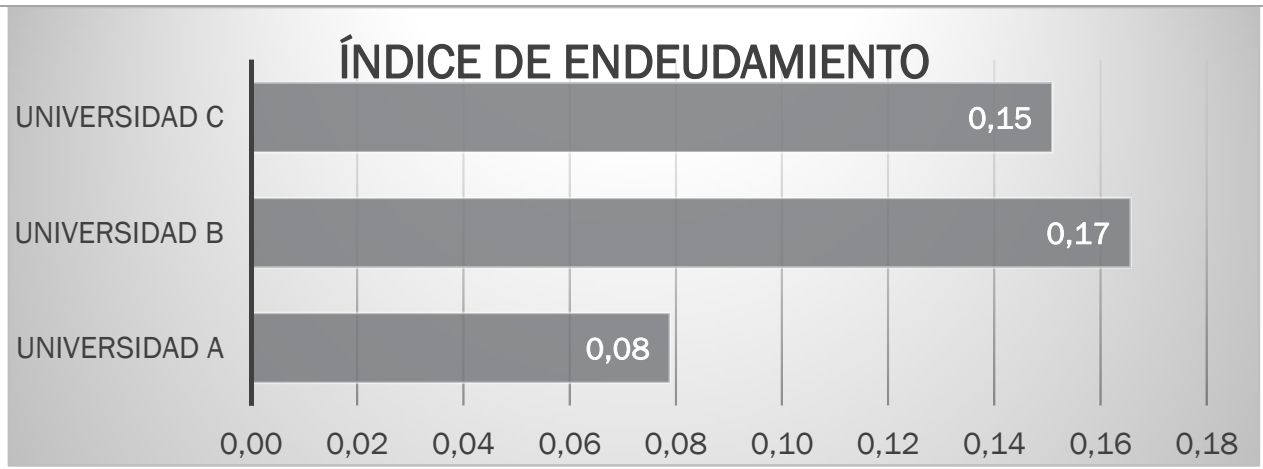

Fuente: Elaborado por los autores.

El nivel de endeudamiento es bajo en cada una de las instituciones educativas analizadas sin embargo la que mayor índice de endeudamiento muestra es la Universidad B que presenta un nivel de deuda de 17 centavos de dólar por cada dólar que posee en sus activos, porcentajes que son manejables, mientras que la de menor endeudamiento es la Universidad A con 8 centavos de dólar por cada dólar en activos.

Figura 8. Comparación del índice de apalancamiento entre instituciones

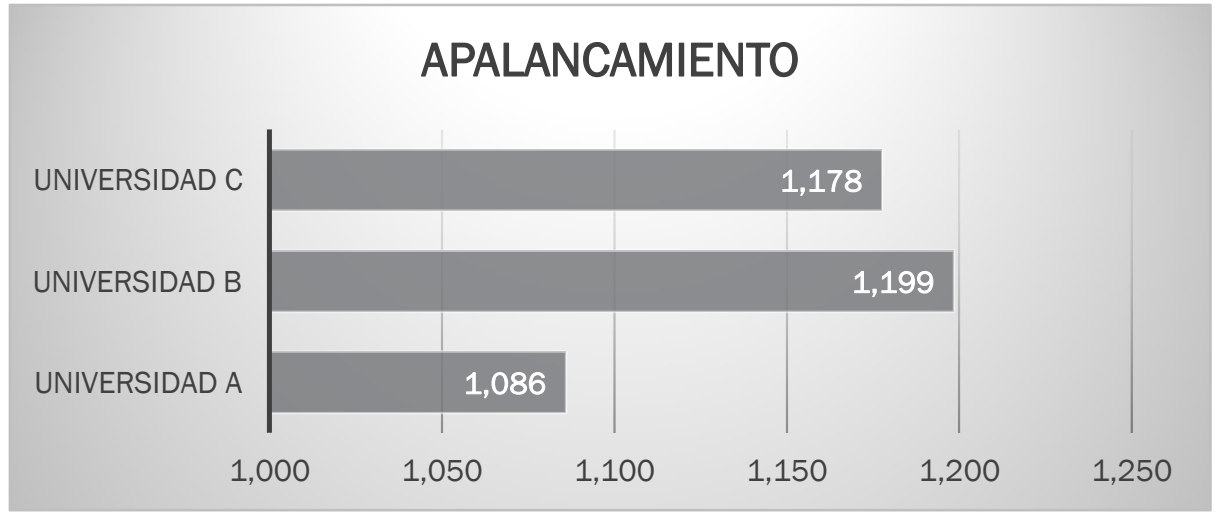

Fuente: Elaborado por los autores 
Análisis comparativo de la gestión financiera de tres Instituciones de Educación Superior del cantón Cuenca

Vol. 2, núm. 1., (2018)

Héctor Alexis Tapia Cárdenas; Carmen Yolanda Jaramillo Calle; Glenda Maricela Ramón Poma; Pedro Yamil Astudillo Arias; Daniel Fernando Cajas Cajas

Este indicador mide la cantidad de unidades monetarias recibidas por cada dólar invertido en el patrimonio, mostrando así un mayor índice la Universidad B, mientras la de menor índice es la Universidad A.

Figura 9. Comparación del índice de firmeza entre instituciones

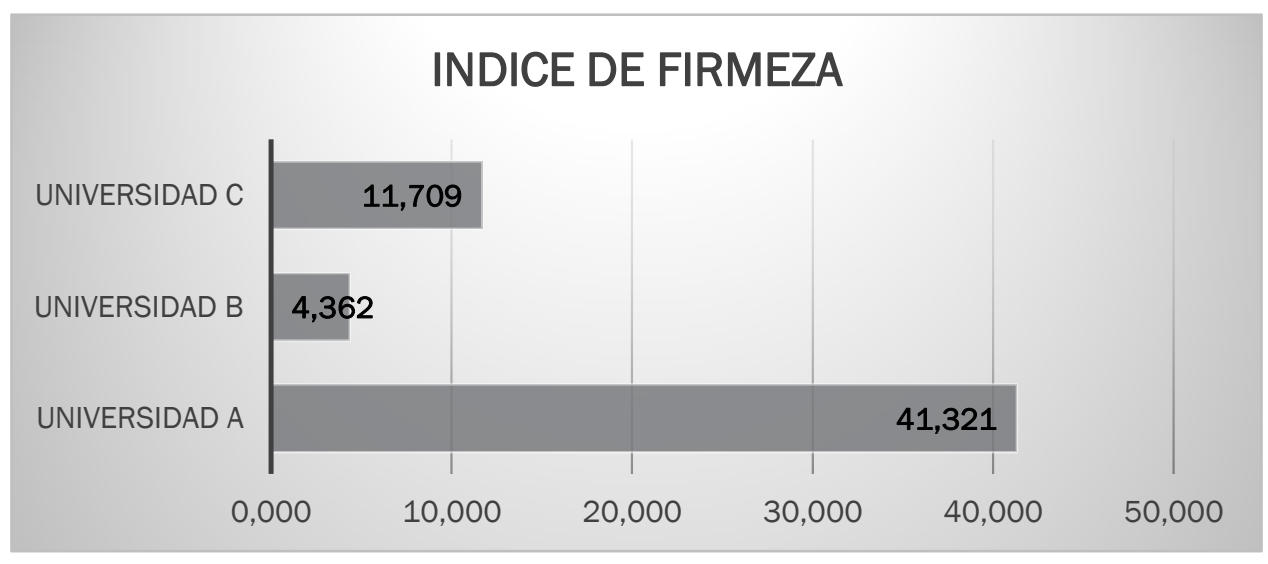

Fuente: Elaborado por los autores

Este indicador muestra la relación del financiamiento de los activos fijos a mayor resultado, lo que nos indicará que los activos han sido financiados principalmente con capitales propios, en este caso la que mejor indicador tiene es la Universidad A mientras la que presenta un menor índice es la Universidad B, sin embargo, su resultado muestra que la principal financiación para sus activos fijos es el capital propio de la institución. 


\section{Análisis comparativo de la gestión financiera de tres Instituciones de Educación Superior del cantón Cuenca}

Vol. 2, núm. 1., (2018)

Héctor Alexis Tapia Cárdenas; Carmen Yolanda Jaramillo Calle; Glenda Maricela Ramón Poma;

Pedro Yamil Astudillo Arias; Daniel Fernando Cajas Cajas

\section{Figura 10. Comparación del Índice Rendimiento sobre Activo entre Instituciones}

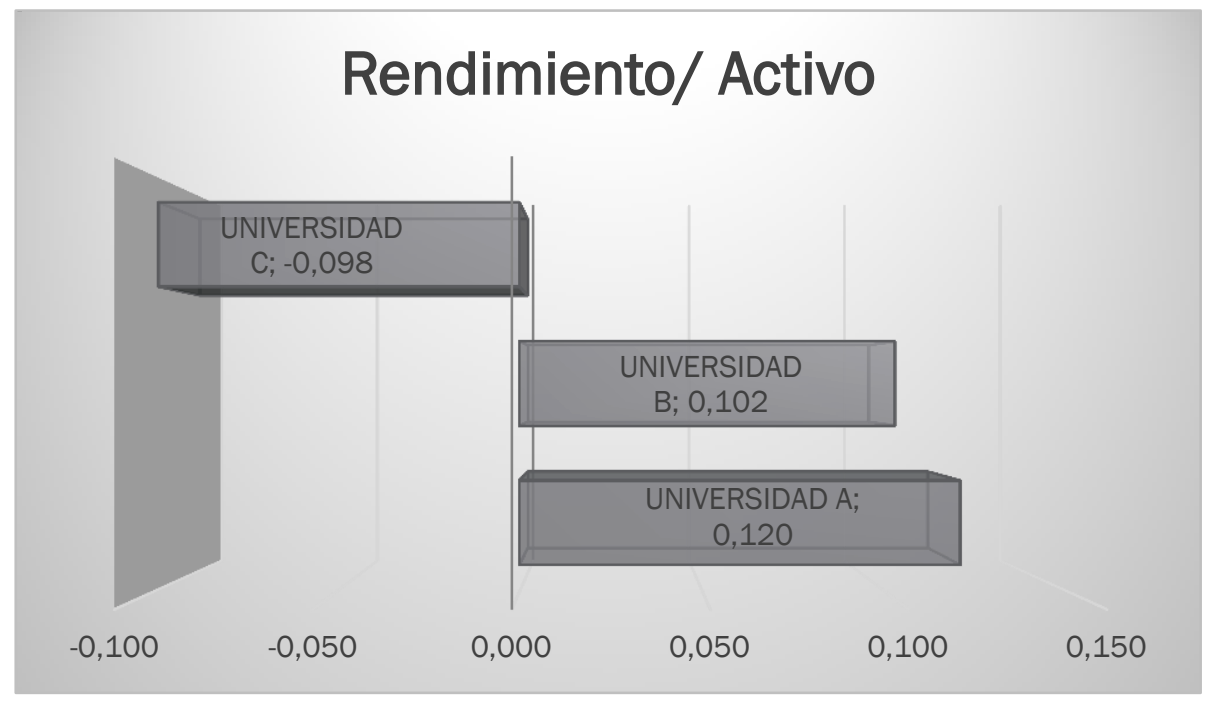

Fuente: Elaborado por el autor.

El rendimiento sobre el activo muestra cuanto produce de utilidad cada dólar invertido en los activos, como podemos apreciar la Universidad A muestra el más alto índice, mientras que la Universidad $\mathrm{C}$ es la que muestra una situación más crítica pues al reflejar perdida en sus utilidades muestra una relación negativa en el resultado del indicador.

\section{Conclusiones}

Luego de haber realizado la investigación de cada una de las instituciones de educación superior y haber realizado las comparaciones respectivas en cuanto se refiere a estudiantes, docentes, sedes y extensiones, y el comportamiento financiero de cada una se ha llegado a las siguientes conclusiones: 


\section{Análisis comparativo de la gestión financiera de tres Instituciones de Educación Superior del cantón Cuenca}

Vol. 2, núm. 1., (2018)

Héctor Alexis Tapia Cárdenas; Carmen Yolanda Jaramillo Calle; Glenda Maricela Ramón Poma; Pedro Yamil Astudillo Arias; Daniel Fernando Cajas Cajas

- Las tres instituciones tienen un gran número de estudiantes estando en primer lugar la Universidad B, luego la Universidad A y por último la Universidad C, a pesar de que la última cuenta con un mayor número de sedes y extensiones, lo que permite visualizar los ingresos que cada una obtiene en cada periodo analizado.

- Luego se ha podido determinar también los docentes con los cuales han trabajado cada institución, en donde la investigación nos da a conocer que la Universidad B tiene un número menor de profesores a pesar de tener más que el doble de estudiantes de la Universidad C y un porcentaje similar de la Universidad A, particular que puede ser sustentado por el número de sedes y extensiones.

- En cuanto al análisis de la situación de las instituciones, podemos determinar que por su capital y nivel de ingresos la institución más grande es la Universidad A, seguido por la Universidad B y por último la Universidad C, mientras que la más importante dado al número de alumnos es la Universidad B seguida por la Universidad A y la Universidad C para finalizar; por otro lado la de mayor presencia es la Universidad C, pues tiene una cobertura mayor que el resto de instituciones.

- La institución más rentable es la Universidad A, pues es la que mayor utilidad generó y la que más rendimiento obtuvo con relación a sus activos y patrimonio seguido de la institución B mientras que la universidad C se ve en una situación desfavorable pues obtuvo una perdida que viene en incremento desde los dos años anteriores al periodo de evaluación, esto se debe al nivel de gasto desproporcionado con relación a los ingresos, 


\section{Análisis comparativo de la gestión financiera de tres Instituciones de Educación Superior del cantón Cuenca}

Vol. 2, núm. 1., (2018)

Héctor Alexis Tapia Cárdenas; Carmen Yolanda Jaramillo Calle; Glenda Maricela Ramón Poma; Pedro Yamil Astudillo Arias; Daniel Fernando Cajas Cajas

pues si los ingresos se reducen los egresos se reducen pero no en igual proporción y si los ingresos aumentan, también aumentan los egresos pero en mayor medida; Mientras que la Universidad B y A mantiene sus egresos por debajo de sus ingresos y si estos varían los egresos también lo hacen pero siempre por debajo de los ingresos, cabe acotar que la principal fuente de egresos por su naturaleza son los sueldos y salarios de docentes, mientras que los principales ingresos son las colegiaturas y matriculas de tercer nivel y las asignaciones corrientes por parte del estado, mientras que los estudios de cuarto nivel no fueron ingresos de mayor representación.

- El nivel de endeudamiento por parte de las instituciones de educación superior es manejable con relación a sus activos, pero la cobertura de intereses es favorable para la Universidad B y para la Universidad A no poseen obligaciones de este tipo mientras que la Universidad C es la que posee inconvenientes de este tipo.

\section{Bibliografía.}

CEAACES. (2013). Informe General sobre la Evaluación, Acreditación, y Categorización de las Universidades y Escuelas Politécnicas. Quito.

CEAACES. (21 de Agosto de 2017). ceaaces.gob.ec. Obtenido de http://www.ceaaces.gob.ec/sitio/acreditacion-y-categorizacion/

Chavéz, J. (2003). Finanzas Teoría Aplicada para Empresas. Quito, Ecuador: Abya Yala.

Córdova Padilla, M. (2016). Análisis Financiero. Bogotá, Colombia: Ecoe Ediciones.

Domínguez, J., \& Olalla, F. (2012). Introducción al análisis financiero. Madrid: Escuela de Organización Industrial. 


\section{Análisis comparativo de la gestión financiera de tres Instituciones de Educación Superior del cantón Cuenca}

Vol. 2, núm. 1., (2018)

Héctor Alexis Tapia Cárdenas; Carmen Yolanda Jaramillo Calle; Glenda Maricela Ramón Poma; Pedro Yamil Astudillo Arias; Daniel Fernando Cajas Cajas

Escobar, M. (22 de agosto de 2013). gestiopolis.com. Obtenido de www.gestiopolis.com/administracion-financiera-y-analisis-financiero-para-la-toma-dedecisiones/.

ESTUPIÑAN, R., \& ESTUPIÑAN, O. (2007). Analisis financierdo y de gestión. Colombia.

León Quinde, F. (2014). Practica Constitucional: Manual Teórico Práctico de Derecho constitucional Ecuatoriano, Derechos y Garantias. Editorial Jurídica Carrión.

Leon, A. (diciembre de 2007). Que es la educación. Mérida, Venezuela.

Muñoz, V. (2012). El Derecho a la Educación: una mirada Comparativa: Argentina Uruguay Chile y Finlandia. El Derecho a la Educación: una mirada Comparativa: Argentina Uruguay Chile y Finlandia. UNESCO- Oficina Regional Para la Educación y en America Latina y el Caribe.

Navas, m. (2009). Análisis financiero: una herramienta clave para una gestión financiera eficiente. Revista Venezolana de Gerencia, vol. 14,, 612.

Pérez E., L. (1993). Un método eficaz para el Análisis Financiero de Pequeños y Medianos Proyectos de Inversión. San José, Costa Rica: IICA.

Rubio, O. (2007). MANUAL DE ANÁLISIS FINANCIERO.

Valls P., J. R. (1999). Análisis de Balances. Madrid: FC Editorial. 